\title{
The Role of the Inhibition of Natural Number Based Reasoning and Strategy Switch Cost in a Fraction Comparison Task
}

\author{
Jo Van Hoof ${ }^{1}$ (D) Eva Ceulemans $^{2}$ (iD), Wim Van Dooren ${ }^{1}$ \\ ${ }^{1}$ Centre for Instructional Psychology and Technology, University of Leuven, Dekenstraat 2, Belgium \\ ${ }^{2}$ Research Group of Quantitative Psychology and Individual Differences, University of Leuven, \\ Tiensestraat 102, 3000 Leuven, Belgium
}

Previous research amply showed the importance of a good fraction understanding but also people's lack of fraction understanding. It is therefore important to investigate the cognitive processes that underlie reasoning with fractions. The present study investigated the role of inhibition and switch costs in fraction comparison tasks. Participants solved a fraction comparison task that alternated between 4 items congruent and 4 items incongruent with natural number reasoning. This allowed to not only investigate congruency switch effects, but also inhibition, given that inhibition was experimentally increased by the prolonged exposure to incongruent trials. Based on data of seventh graders, the present study showed that inhibition does not only play a role in learners' general mathematics achievement, but also in specific areas of mathematics, such as fractions. Moreover, a switch cost was found in the lower accuracy rates and higher reaction times needed to correctly solve switch items compared to non-switch items.

Key words: fraction, rational numbers, switch cost, inhibition

\section{Introduction}

\section{Fraction Understanding}

The acquisition of a good understanding of fractions is of crucial importance for learners' mathematical development (Bailey, Hoard, Nugent, \& Geary, 2012; Booth, Newton, \&
Twiss-Garrity, 2014; Siegler et al., 2012). It forms the basis for a good understanding of later mathematical contents, such as algebra, proportional reasoning, probability, and calculus (see for example Behr, Lesh, Post, \& Silver, 1983; Booth et al., 2014). It is therefore worrying that during the last decades, research amply showed that learning fractions is challenging for many learners (see

Correspondence concerning this article should be addressed to Jo Van Hoof, Centre for Instructional Psychology and Technology, University of Leuven, Dekenstraat 2, Belgium. E-mail: jo.vanhoof@kuleuven.be

Received January 17, 2020 
for example Gabriel et al., 2013; Moss, 2005; Siegler et al., 2012).

\section{The Natural Number Bias}

In the literature, several reasons can be found why fractions are so hard to grasp for learners. The fractions' conceptually different meanings need to be understood: fractions as quotient, ratio, operator, and measure; learners need to develop a concept of number that is based on multiplicative relations instead of additive ones, etc. (for an overview, see Moss, 2005). Remarkably, one reason received a lot of research attention in the last two decades, namely learners' tendency to apply natural number properties to rational numbers. This phenomenon is known as the natural number bias (for an overview, see Van Hoof et al., 2017). The natural number bias has frequently been found in participants' higher accuracy rates and faster reaction times to correctly solve congruent fraction comparison items (where natural number reasoning leads to a correct answer, for example, which number is larger: $2 / 5$ or $7 / 9 ; 2 / 5$ is smaller than $7 / 9$; just like 2 is smaller than 7 and 5 is smaller than 9) compared to incongruent fraction comparison items (where natural number reasoning leads to an incorrect answer, for example, which number is larger: $2 / 5$ or $7 / 29 ; 2 / 5$ is larger than $7 / 29$, while 2 is smaller than 7 and 5 is smaller than 29). Previous studies showed that (traces of) a natural number bias could not only be found in elementary-school children, but also in secondary school children, adults, and even prospective teachers (Van Hoof et al., 2017; Behr et al., 1984; Clarke \& Roche, 2009; Depaepe et al., 2013; McMullen, Laakkonen, Hannula-Sormunen, \& Lehtinen, 2015; Vamvakoussi \& Vosniadou, 2010). Importantly, several longitudinal studies found that a good understanding of the numerical magnitude of fractions is of crucial impor- tance. It forms a first step and is necessary to understand other aspects of fractions, such as doing operations with fractions (Van Hoof et al., 2018; McMullen et al., 2015; McMullen \& Van Hoof, 2020).

However, many learners have the misconception that the numerical value of a fraction increases when its numerator, denominator, or both increase.

\section{The Role of Intuitive Processing}

Mistakes on fraction tasks do not only occur when learners have a wrong understanding of fractions. Even when learners have acquired a good understanding of fractions, they can still make mistakes on fraction tasks, due to the intuitive processing that is assumed to take place. This can be explained by the dual process theory of reasoning, which has been proven to describe the intuitive nature of incorrect reasoning in several mathematical domains (Gillard et al., 2009), including fractions (DeWolf \& Vosniadou, 2015). The dual process theory argues that people have two different reasoning systems: an intuitive one (which is fast, automatic, and undemanding of working memory), and an analytical one (which is slow, deliberate, and demanding of working memory). Intuitive reasoning is engaged in by default, and often leads to accurate responses (for example in congruent items). However, in some situations, it leads to an incorrect response and analytical reasoning is needed. With regard to fractions, learners are influenced by their intuitive natural number based concept of number (including fractions), which needs to be overcome in incongruent fraction tasks. In this light, evidence for a natural number bias has also been shown in learners' reaction time data. More specifically, longer reaction times are found on correctly solved incongruent items compared to correctly solved congruent ones, 
given that analytical reasoning is needed in the former.

Based on the dual process theory, an incorrect response to a task can therefore be the result of two possible situations. First, it is possible that an incorrect intuitive thought process occurs, without intervention of the analytical reasoning system. Second, it is possible that the analytical reasoning system does intervene, evaluates the intuitive answer, tries to inhibit it, but fails to generate the correct answer (Gillard et al., 2009). The latter implies that inhibition might be of big importance in learners' fraction understanding, and more precisely when solving incongruent fraction tasks.

\section{Inhibition}

Inhibition has been described as "the ability to ignore information or responses that are irrelevant to the task at hand" (Gilmore, Göbel, \& Inglis, 2018). Recently, there is a growing and promising research interest in the field of cognitive psychology in the role of inhibitory control as an important part of learners' cognition and development. Also, in the area of mathematical thinking and learning, inhibition has been shown to play an important role in learners' mathematical performance (see for example Van Dooren \& Inglis, 2015). At the moment, however, the research field has two limitations. First, the role of inhibitory control has mostly been investigated in relation to mathematics achievement in a general sense. Therefore, research is needed to investigate the role of inhibitory control to specific components of mathematics, such as fraction understanding, and try to unravel the role of inhibition in very specific processes, for instance comparing the magnitude of fractions (Van Dooren \& Inglis , 2015; Gilmore et al., 2018). Second, most of the studies linking inhibitory control with (certain aspects of) mathematics achievement are correlational in nature; research showing the causal relation between inhibition and mathematics achievement is still missing. As stated by previous research, "a limitation of such a correlational approach is that none of the classical inhibitory control tasks assess individual differences in the ability to inhibit a misleading strategy" (Rossi, Vidal, Letang, Houdé, \& Borst, 2019, p. 24).

The only study, as far as we know, that investigated in a causal way the need for inhibition when comparing fractions is the recent study of Rossi et al. (2019). In this study, a negative priming paradigm was used to investigate the role of inhibition in fraction comparison tasks. The reasoning of a negative priming paradigm is that if a strategy or distractor is inhibited on a given item (referred to as a prime), this will lead to a decrease of that strategy/distractor on the item following the prime (referred to as the probe) compared to the control condition where inhibition of that strategy/distractor is not needed in the prime. This will lead to lower accuracy rates and/or higher reactions times on a probe (where the inhibited strategy on the prime is needed to come to the correct solution) following a prime compared to the same type of probe following a control item. Rossi et al. (2019) showed this with $9^{\text {th }}$ graders and adults, who compared fractions with common denominators (congruent fraction comparison tasks; e.g., 3/7 vs. 5/7). They needed more time to do this after they compared fractions with common numerators (incongruent fraction comparison tasks in which the strategy "the larger the natural number, the larger the fraction" needed to be inhibited; e.g., $7 / 2$ vs. $7 / 8$ ) than after they had to indicate which of two fractions had a larger denominator compared to its numerator (neutral task in light of natural number bias). This result shows that inhibition of "the greater the natural number, the greater the fraction" reasoning takes place when correctly comparing fractions with common numera- 
tors. However, as stated by Rossi et al. (2019), there were several limitations in this study. First, in the control trials the prime consisted of a different task (indicate which fraction out of two has a larger denominator in comparison with its numerator), while in the test trials the prime consisted of the same task as the probe (indicate which fraction out of two has the largest numerical value). Therefore, the negative priming effect could be explained by the difference between a task switch cost in the control trials and a strategy switch cost in the test trials. Second, participants received 24 practice trials accompanied with feedback (regarding the correctness of the answer), with the aim to familiarize the participants with the task. By providing feedback, it might be possible that there was a learning effect and participants changed in their choice of strategy to solve the fraction comparison tasks. Third, the fractions used in the fraction comparison task had several limitations. For example, only single digit numerators and denominators were used. As stated by Rossi et al. (2019), this might have led participants to rely more on the "the greater the whole number, the greater the fraction" heuristic, given that previous research concluded that the use of two digit fractions led to an increase of holistic processing of fractions (Rossi et al., 2019; Schneider \& Siegler, 2010). Taken together, Rossi et al. (2019) suggest that "future studies should investigate whether similar effects can be observed when different prime items are designed in the control trials, when no feedbacks are provided in the practice trials, and when a wider range of numbers including two digits number is used to generate the fractions" (Rossi et al., 2019, p. 30). These issues will be addressed in the current study. However, we will use a slightly different methodology compared to the negative priming paradigm, allowing us to not only investigate the role of inhibition in fraction comparison tasks, but also the role of a switch cost.

\section{Strategy Switch Cost}

A phenomenon that gained increased research interest is the strategy switch cost. Next to the widely known task switch cost (e.g., Verbruggen, Liefooghe, \& Vandierendonck, 2006), recent literature has focused on and investigated the strategy switch cost, which has been defined as "the time taken by active control processes to reconfigure the cognitive system for the execution of another strategy. More specifically, when a problem is solved with a given strategy, and the next problem has to be solved with another strategy, the cognitive system must be reconfigured." (Schillemans, Luwel, Onghena, \& Verschaffel, 2011, p. 25). This reconfiguration comes with a cost, which has repeatedly been found in previous research in participants' longer reaction times and lower accuracy rates on switch compared to non-switch trials. This is no surprise given that the reconfiguration of the cognitive system relies on the three main executive functions: the previous strategy must be inhibited, attention must be shifted from the 'old' strategy to the 'new' strategy, and procedures of the 'new' strategy must be retrieved by the working memory (e.g., Schillemans et al., 2011). Interesting to note is that there is often an asymmetrical switch cost found in a counter-intuitive direction, namely a larger cost going from a difficult to an easier task than the reverse (see for example Luwel et al., 2009). Allport et al. (1994) give the following explanation, which is in line with the negative priming paradigm: when solving a difficult, incongruent task, the intuitive reasoning as used to solve an easy congruent task must be inhibited to come to the correct answer. This 'inhibition mode' carries over to the next task. Therefore, if the next task is an easy congruent one, an extra inhibition (of the previous inhibition) is needed, leading to larger switch costs. 
Since the strategy switch cost has solely been studied, as far as we know, in numerosity judgment (Schillemans et al., 2011), computational estimation, and two-digit addition tasks (both by Lemain \& Lecacheur, 2010), further research is needed to investigate whether the same strategy switch cost is also present in different aspects of the mathematical curriculum (Schillemans et al., 2011), such as fractions. Applied to fraction comparisons, when fractions are compared in a holistic way, every fraction comparison task is the same and congruency has no effect on the mental processes needed to solve a fraction comparison task. However, when participants compare fractions in a componential way (and therefore the natural number bias can play a role), congruency does have an effect. While congruent items can be correctly solved by focusing on the natural number components, a different strategy is needed to correctly solve incongruent fraction comparison tasks. This switch between strategies can therefore lead to a switch cost. In line with Schmidt and Houwer (2011), we will refer to this cost as a congruency switch cost. This describes the phenomenon that when the congruency of a trial is the same as the previous trial, this will incur a benefit (higher accuracy rates and lower response times) compared to the situation in which the previous trial has the opposite congruency.

\section{The Present Study}

In the present study, we investigated both the causal role of inhibition in fraction comparison tasks and whether a switch cost could be replicated when participants solve a fraction comparison task. In total, the present study addressed four research questions. First, we investigated whether our study would confirm previous research by finding traces of the natural number bias in learners' accura- cy and/or reaction time data. Our hypothesis was that the participants would have higher accuracy rates on congruent compared to incongruent items and that they would need more time to accurately answer an incongruent item compared to a congruent item. Second, we investigated whether inhibition (of natural number reasoning) is needed when learners compare fractions. Our hypothesis was that inhibition does play a role and this could be found in the higher accuracy rates and lower reaction times needed to correctly solve incongruent trials when inhibition is experimentally increased (by a prolonged exposure to incongruent trials; see method section). Third, we looked whether a congruency switch cost could be found when participants solve a fraction comparison test. Our hypothesis was that, in line with Schmidt and Houwer (2011), we would find a congruency switch effect in the lower accuracy rates and/ or higher reaction times to correctly solve switch items compared to non-switch items. Fourth, if a congruency switch cost was found, we would investigate whether this switch cost was asymmetrical. In line with previous research on other tasks (see above), we hypothesized that if there was an asymmetrical switch cost, switch costs would be larger going from an incongruent to a congruent item than vice versa.

\section{Method}

\section{Participants}

In total, data of $1367^{\text {th }}$ grade students from two different middle-sized schools were collected. We chose to collect data in $7^{\text {th }}$ graders since in this age group we could still expect traces of the natural number bias as well as find individual differences in learners' fraction understanding (see for example Van Hoof et al., 2017). Put differently, we expected that 
the majority of learners would not show ceiling or floor effects in their fraction understanding. Both students from general education ( $n=109$ students; 54 female and 55 male, age: $M=12.43$ years, $S D=.64$ ) and from vocational education ( $n=26,19$ female and 7 male, age: $M=12.56, S D=.56$ ) were included. Parents of all the participants signed an informed consent. Data were collected according to the ethical guidelines of the KU Leuven.

\section{Procedure}

Data collection took place in a separate classroom in groups of 8 participants. Learners solved a fraction comparison task in E-Prime.
At the beginning of the task, the students were informed that they would have to choose, as accurately and as fast as possible, the larger fraction out of two by pressing the corresponding key on the keyboard (" $f$ " for left and "j" for right).

\section{Design}

All participants solved a fraction comparison task. Every trial started by a fixation cross (1000 ms) followed by two fractions that were shown simultaneously on a computer-screen (see Figure 1 for item list). All comparison items were displayed as black digits on a white background.

\begin{tabular}{|c|c|c|c|}
\hline Block & Type & Item 1 & Item 2 \\
\hline \multirow[t]{8}{*}{5} & C & $3 / 19$ & $5 / 19$ \\
\hline & C & $21 / 58$ & $25 / 58$ \\
\hline & C & $41 / 65$ & $48 / 65$ \\
\hline & C & $59 / 79$ & $51 / 79$ \\
\hline & IC & $5 / 21$ & $5 / 16$ \\
\hline & IC & $38 / 53$ & $38 / 59$ \\
\hline & IC & $4 / 21$ & $4 / 25$ \\
\hline & IC & $39 / 50$ & $39 / 44$ \\
\hline \multirow[t]{8}{*}{6} & C & $9 / 11$ & $6 / 11$ \\
\hline & C & $32 / 83$ & $38 / 83$ \\
\hline & C & $39 / 51$ & $28 / 51$ \\
\hline & C & $24 / 67$ & $31 / 67$ \\
\hline & IC & $23 / 74$ & $23 / 68$ \\
\hline & IC & $71 / 97$ & $71 / 91$ \\
\hline & IC & $41 / 62$ & $41 / 68$ \\
\hline & IC & $31 / 90$ & $31 / 99$ \\
\hline \multirow[t]{8}{*}{7} & $C$ & $16 / 43$ & $20 / 43$ \\
\hline & C & $16 / 23$ & $12 / 23$ \\
\hline & $C$ & $22 / 39$ & $26 / 39$ \\
\hline & C & $20 / 77$ & $30 / 77$ \\
\hline & IC & $65 / 81$ & $65 / 76$ \\
\hline & IC & $5 / 6$ & $5 / 9$ \\
\hline & IC & $22 / 51$ & $22 / 47$ \\
\hline & IC & $40 / 87$ & $40 / 77$ \\
\hline \multirow[t]{8}{*}{8} & $C$ & $48 / 69$ & $40 / 69$ \\
\hline & C & $6 / 17$ & $3 / 17$ \\
\hline & C & $29 / 44$ & $35 / 44$ \\
\hline & $C$ & $15 / 38$ & $11 / 38$ \\
\hline & IC & $48 / 89$ & $48 / 95$ \\
\hline & IC & $29 / 68$ & $29 / 74$ \\
\hline & IC & $49 / 58$ & $49 / 55$ \\
\hline & IC & $21 / 25$ & $21 / 29$ \\
\hline
\end{tabular}

Figure 1 Item list of the fraction comparison task. 
Since previous research warned for the possible undesired learning effect of adding many practice trials (see for example Rossi et al., 2019), only four practice trials with feedback were included in the experiment. This way, we tried to avoid an undesired learning effect, but still made sure the participants understood the task. The test itself contained 32 congruent (fractions with common denominator) and 32 incongruent items (fractions with common numerator), allowing to investigate the role of a natural number bias. In total, eight blocks of eight items were used. Each block consisted of first four congruent items followed by four incongruent items. By ordering the items in such a way that four congruent items were presented in a row, and then four incongruent items in a row, we were able to experimentally increase participants' state of inhibition: a prolonged exposure to incongruent trials might increase participants' inhibition. Therefore, we compared participants' performances on every second and fourth incongruent trial. We decided not to use the first incongruent trial for these comparisons, since this item is a switch trial and might be affected by a congruency switch (too). With regard to the congruency switch effect, due to the design of our fraction comparison task (each block consisted of first four congruent items followed by four incongruent items), we could distinguish 15 switch tasks ( 7 from incongruent to congruent and 8 from congruent to incongruent) and 16 stay tasks (see Figure 2). This distinction between switch tasks and stay tasks made it possible to investigate a congruency switch effect and the possible asymmetrical nature of this effect.

As stated above, it is necessary to control the item list in a fraction comparison task very strictly to prevent unwanted strategies to occur, such as gap thinking or benchmarking (see for example Gonzalez-Forte et al., 2019). Therefore, the fraction comparison task was controlled for benchmarking to 0 , $1 / 2$, and 1 . This was done by making sure that the numerical value of the fractions was never smaller than 0.1 or larger than 0.9. Moreover, in every set of four comparison items, in two fraction pairs both fractions were above one half $(A)$ and in two pairs both were below one half $(B)$, leading to six possible combinations, which were - as much as possible - equally represented: $A A B B, A B A B, A B B A$, $B B A A, B A A B, B A B A$. Further, within each set of four comparison items, the largest fraction appeared twice on the right side and twice on the left side of the screen. Next, no numerator or denominator was a multiple of the other fractions' numerator of denominator, to avoid the strategy of simply multiplying a component. Further, all fractions were below 1, every numeral from 0 until 9 appears equally often in the trials and no simplifiable fractions were included. At last, two versions of the fraction comparison were used. In the second version the first and last item of each set were swapped, preventing that certain item characteristics may have any influence on the switch cost.

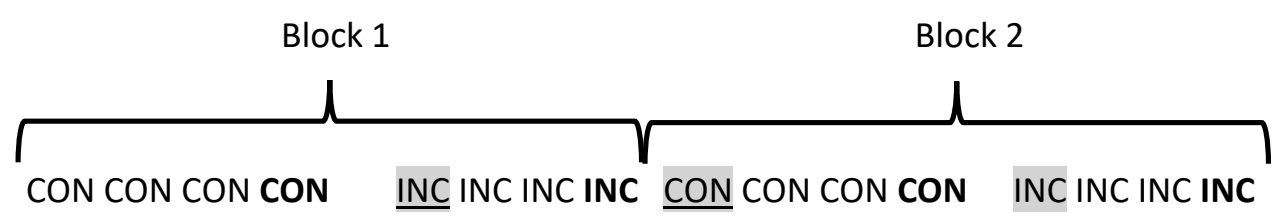

Figure 2 Design of the fraction comparison test ( 2 blocks out of 8 ). Switch items are highlighted and underlined, stay items are bold, the rest are control items. 
The data that support the findings of this study are publicly available at OSF (https:// osf.io/zcx9g/).

\section{Results}

To be able to see a congruency switch effect, it was necessary that the participants understood the task and were able to solve both congruent and incongruent fraction comparison tasks. Therefore, participants who did not score above $50 \%$ accuracy on both the congruent and incongruent control items were not included in the further data analyses. In total $337^{\text {th }}$ graders were excluded ( $=24.26 \%$ of the data).

Moreover, with regard to participants' reaction time, we controlled whether all trials had a reaction time of at least $500 \mathrm{~ms}$. Based on our previous studies, we assumed that it is impossible for participants to process both fractions and compare them in less than $500 \mathrm{~ms}$. Therefore, reaction times below $500 \mathrm{~ms}$ are likely to represent a pressing mistake. Further, for each individual participant, trials that were three standard deviations away from the mean were deleted as outliers. In total, $2.14 \%$ of the dataset was deleted.

Since our data had a repeated measures design, we conducted a Generalized Estimation of Equations (GEE) in order to correct for repeated (and probably correlated) measures within participants (Liang \& Zeger, 1986).

\section{Natural Number Bias}

\section{Accuracy data}

First, we looked at learners' accuracy data by comparing learners' mean score on congruent versus incongruent items. Given the dichotomous nature of participants' accuracy levels, a logistic regression model was run. A significant main effect was found of congru- ency, Wald $X^{2}(1, N=6393)=36.75, p<.001$; $O R=1.90,95 \% \mathrm{Cl}[1.60,2.26]$, indicating that participants' mean accuracy scores were significantly higher on congruent (mean score = $.93, S D=.25$ ) versus incongruent items (mean score $=.88, S D=.33$ ).

\section{Reaction time data}

Second, we looked at learners' reaction time data by comparing learners' mean time needed to correctly solve congruent versus incongruent items. Given the continuous nature of participants' reaction time, a linear regression model was run. A significant main effect of congruency was found, Wald $X^{2}(1, N=5775)$ $=31.30, p<.001, d=.55$, showing that learners needed significantly more time to correctly solve incongruent $($ mean $=3039 \mathrm{~ms}, S D=$ 2097) compared to congruent items (mean = $2588 \mathrm{~ms}, S D=1642$ ).

\section{Inhibition}

\section{Accuracy data}

First, we looked at learners' accuracy data by comparing learners' mean score on the second and fourth incongruent trial. A significant main effect was found of item place, Wald $X^{2}(1$, $N=1586)=10.99, p<.001 ; O R=1.55,95 \%$ $\mathrm{Cl}[1.13,2.11]$, indicating that participants' mean accuracy scores were significantly higher on the fourth (mean score $=.91, S D=.29$ ) compared to the second incongruent trial (mean score $=.86, S D=.35$ ).

\section{Reaction time data}

Second, we looked at learners' reaction time data by comparing learners' mean time needed to correctly solve the second versus the fourth incongruent item. A significant main effect of place item was found, Wald $X^{2}(1$, 
$N=1401)=30.06, p<.001, d=.55$, showing that learners needed significantly less time to correctly solve the fourth (mean $=2753 \mathrm{~ms}$, $S D=1798$ ) compared to the second incongruent item ( mean $=3243 \mathrm{~ms}, S D=2407)$.

\section{Congruency Switch Cost}

Given that we were interested in comparing learners' performances in switch versus nonswitch items, in the following analyses, only the data on the switch and non-switch items were included.

\section{Accuracy data}

To investigate whether a congruency switch could be found in participants' accuracy data, we compared the scores on switch items with those on non-switch items. A significant main effect was found of switching, Wald $X^{2}(1, N=$ $3105)=8.17, p<.01 ; O R=2.02,95 \% \mathrm{Cl}[1.59$, 2.58], indicating that participants' mean accuracy scores were significantly higher on nonswitch (mean score $=.93, S D=.26$ ) versus switch items (mean score $=.87, S D=.34$ ). In a next step, we looked whether this congruency effect had an asymmetrical nature. No significant interaction effect was found between switching and congruency, Wald $X^{2}(1, N=$ $3105)=0.03, p=.86$, indicating that the congruency switch cost in participants' accuracy scores was equally large going from incongruent to congruent items as vice versa.

\section{Reaction time data}

To investigate whether a congruency switch could be found in participants' reaction time data, we compared the reaction times participants needed to correctly solve switch items versus non-switch items. A significant main effect of switching was found, Wald $X^{2}$ (1, $N=2790)=14.70, p<.001, d=.38$, showing that learners needed significantly more time to correctly solve switch items (mean $=3068 \mathrm{~ms}, S D=$ 1891) compared to non-switch items (mean = 2543 ms, SD = 1678). Moreover, no significant interaction effect was found between switching and congruency, Wald $X^{2}(1, N=2790)=.87$, $p=.35$, showing that the congruency switch cost in participants' reaction times was equally large going from incongruent to congruent items as vice versa.

\section{Discussion}

Given that previous research repeatedly showed the high importance of a good fraction understanding, but at the same time people's lack of fraction understanding, it is important to investigate the cognitive processes that underlie reasoning with fractions. More precisely, the present study had two main aims. Our first aim was to provide causal evidence for the role of inhibition, specifically in fraction comparison tasks. Until now, the role of inhibitory control has mostly been investigated in relation to mathematics achievement in a general sense, rather than to specific components of mathematics, such as fraction understanding (Van Dooren \& Inglis, 2015; Gilmore et al., 2018). Moreover, most of the studies are correlational in nature; research showing the causal relation between inhibition and mathematics achievement is still missing.

With regard to fraction comparison tasks, starting from a dual process perspective, it is suggested that when people have the acquired knowledge to solve fraction tasks, an incorrect response to a fraction task can be explained by two different situations. First, it is possible that people incorrectly reason in an intuitive natural number based way without the intervention of the analytical reasoning system. Second, it is possible that the analytical reasoning system does intervene, tries to inhibit the intuitive an- 
swer, but fails to generate the correct answer (e.g., Gillard et al., 2009). The latter implies that inhibition might play a role in learners' fraction understanding, and especially when solving fraction tasks that are incongruent with natural number reasoning.

As far as we know, there is only one study of Rossi et al. (2019) that investigated the role of inhibition in fraction tasks in a causal way, using a negative priming paradigm. This study concluded that both in adolescents and adults the inhibition of natural number based reasoning is needed to correctly compare fraction comparison task with common components. In the present study, we tried to replicate their findings while addressing the several shortcomings of this study.

The second aim of the present study was to investigate whether a switch cost could be replicated when participants solve a fraction comparison task. Given that strategy switch costs have solely been investigated (to the best of our knowledge) in numerosity judgment, computational estimation, and two-digit addition tasks, there was a need to investigate whether similar switch costs could be found in different aspects of mathematics, such as fraction understanding. In line with Schmidt and Houwer (2011), we referred to this switch cost as a congruency switch cost.

In total, the present study addressed four research questions. First, we investigated whether our study would confirm learners' struggle to understand fractions by finding traces of the natural number bias in learners' accuracy and/or reaction time data. A clear indication for the natural number bias was found both in participants' accuracy levels (significantly higher on congruent compared to incongruent items) and in their reaction time data (significantly more time to correctly answer incongruent compared to congruent items). Second, by experimentally increasing a state of inhibition, we investigated wheth- er inhibition (of natural number reasoning) is needed when learners compare fractions. Results indicated higher accuracy rates and lower reaction times needed to correctly solve incongruent trials when inhibition is experimentally increased. This result adds to the current literature by showing that inhibition does not only play a role in learners' general mathematics achievement, but also in specific areas of the mathematics curriculum, such as fractions, and more specifically comparing the size of fractions where the straightforward application of natural number knowledge would lead to the wrong conclusion. Third, we looked whether a congruency switch cost could be found when participants solve a fraction comparison test. A congruency switch effect was found in the lower accuracy rates and higher reaction times to correctly solve switch items compared to non-switch items. Fourth, if a congruency switch cost was found, we would investigate whether this switch cost was asymmetrical. The results found no asymmetrical switch cost.

The present study does not only add new knowledge to the research field on strategy switch costs by finding that switch costs also occur in fraction tasks, but it also addresses the current debate in the literature on whether people process fractions in a componential or holistic way. The results of the present study suggest that adolescents compare fractions in a componential way. If fractions were compared in a holistic way, congruency would have no effect on the mental processes needed to solve a fraction comparison task. However, we found that congruency does have an effect, and this is also the case when fractions are compared in a componential way, given that a different strategy is needed to correctly solve incongruent/congruent fraction comparison tasks. As stated by Obersteiner et al. (2013), it is important to note that "the activated mental representations of fractions are 
likely to depend on the specific fractions being processed. Fraction comparison problems that involve fraction pairs with common components can be solved more efficiently without activating holistic magnitude representations for the fractions." (Obersteiner et al., 2013, p. 65). Therefore, it might be possible that different results can be found in fractions without common components. This should be investigated by future research. Moreover, while our results suggest that adolescents use componential reasoning, these data should be supplemented with interview data to confirm that the same strategies are also found in participants' verbal reports when describing how they solve fraction comparison tasks. Moreover, future research should investigate whether similar results can be found in other age groups. Next to the fact that inhibition skills mature (e.g., Gilmore, Keeble, Richardson, \& Cragg, 2015), participants' level of expertise with the task (in our case fraction comparisons) can also play a role in the involvement of inhibitory processes (see for example Cragg \& Gilmore, 2014).

Further, future research should investigate the similarities and differences between congruency switch, strategy switch, and task switch costs. For example, a big difference between congruency and strategy switch on the one hand, and task switch on the other hand, is that in task switching people need to change their goal setting, while this is not the case in the congruency/strategy switch tasks. In the latter, the task and therefore the goal remains the same between trials, the only difference is the way of reaching that goal. Therefore, it might be possible that the degree in which executive control functions, and more specifically shifting, is different in task switching assignments, compared to congruency/strategy switch tasks (see for example Luwel et al., 2013).

The present study also has an important educational implication by showing that inhibition plays a role in learners' fraction understanding. Given the crucial importance of fraction understanding for later mathematical development, this study calls for the need to train learners' inhibition skills. Diamond (2013) does not only show that executive functions can be trained, but also that they can be improved at any age level. Important to note is that it has been shown that teachers (especially in the beginning of their teaching career) are not aware of the importance of executive functions for the learning of mathematics (Gilmore \& Cragg, 2014). Therefore, our study suggests that teachers and practitioners should be made aware of the important role of executive functions (such as inhibition) for the learning of fractions and mathematics in general (see also for example Cragg \& Gilmore, 2014).

Moreover, since a congruency switch cost has been found, the present study suggests that it is of importance to pay attention to the precise order in which fraction tasks are handled in the classroom and to provide feedback on the influence of the task characteristics for learners' strategy choice when dealing with fractions (see for example Schillemans, Luwel, Onghena, \& Verschaffel, 2011).

\section{Acknowledgements}

Jo Van Hoof holds a post-doctoral fellowship of the Research Foundation-Flanders (FWO). We would like to thank Stéfanie Vanneste for her help in data collection. The datasets generated during and/or analyzed during the current study are available from the corresponding author on reasonable request.

\section{Authors' ORCID}

Jo Van Hoof

https://orcid.org/0000-0003-4752-6221

Eva Ceulemans

https://orcid.org/0000-0002-7611-4683

Wim Van Dooren

https://orcid.org/0000-0001-5002-4340 


\section{References}

Allport, A., Styles, E. A., \& Hsieh, S. (1994). Shifting intentional set: Exploring the dynamic control of tasks. In C. Umilta \& M. Moscovitch (Eds.), Attention and performance XV (pp. 421-452). Cambridge, MA: MIT Press.

Bailey, D. H., Hoard, M. K., Nugent, L., \& Geary, D. C. (2012). Competence with fractions predicts gains in mathematics achievement. Journal of Experimental Child Psychology, 113(3), 447-455. https://doi.org/10.1016/i.jecp.2012.06.004

Behr, M. J., Lesh, R., Post, T., \& Silver, E. (1983). Rational number concepts. In R. Lesh \& M. Landau (Eds.), Acquisition of mathematics concepts and processes (pp. 91-125). New York, NY: Academic Press.

Booth, J. L., Newton, K. J., \& Twiss-Garrity, L. K. (2014). The impact of fraction magnitude knowledge on algebra performance and learning. Journal of Experimental Child Psychology, 118, 110118. https://doi.org/10.1016/i.jecp.2013.09.001

Cragg, L., \& Gilmore, C. (2014). Skills underlying mathematics: The role of executive function in the development of mathematics proficiency. Trends in Neuroscience and Education, 3(2), 6368. https://doi.org/10.1016/i.tine.2013.12.001

DeWolf, M., \& Vosniadou, S. (2015). The representation of fraction magnitudes and the whole number bias reconsidered. Learning and Instruction, 37, 39-49. https://doi.org/10.1016/i.learninstruc. 2014.07.002

Diamond, A. (2013). Executive functions. Annual Review of Psychology, 64, 135-168. https://doi. org/10.1146/annurev-psych-113011-143750

Gabriel, F., Coché, F., Szucs, D., Carette, V., Rey, B., \& Content, A. (2013). A componential view of children's difficulties in learning fractions. Frontiers in Psychology, 4, 1-12. https://doi.org/10.3389/ fpsyg.2013.00715

Gillard, E., Van Dooren, W., Schaeken, W., \& Verschaffel, L. (2009). Dual processes in psychology of mathematics education and cognitive psychology. Human Development, 52(2), 95-108. https://doi.org/10.1159/000202728

Gilmore, C., Göbel, S. M., \& Inglis, M. (2018). An introduction to mathematical cognition. Routledge.
Gilmore, C., \& Cragg, L. (2014). Teachers' understanding of the role of executive functions in mathematics learning. Mind Brain and Education, 8(3), 132-136. https://doi.org/10.1111/ mbe. 12050

Gilmore, C., Keeble, S., Richardson, S., \& Cragg, L. (2015). The role of cognitive inhibition in different components of arithmetic. ZDM, 47, 771-782. https://doi.org/10.1007/s11858-014-0659-y

González-Forte, J. M., Fernández, C., Van Hoof, J., \& Van Dooren, W. (2019). Various ways to determine rational number size: An exploration across primary and secondary education. European Journal of Psychology of Education, 35, 549-565. https://doi.org/10.1007/s10212-019-00440-w

Lemaire, P., \& Lecacheur, M. (2010). Strategy switch costs in arithmetic problem solving. Memory \& Cognition, 38, 322-332. https://doi. org/10.3758/MC.38.3.322

Luwel, K., Schillemans, V., Onghena, P., \& Verschaffel, L. (2009). Does switching between strategies within the same task involve a cost? British Journal of Psychology, 100(4), 753-771. https://doi. org/10.1348/000712609X402801

McMullen, J., Laakkonen, E., Hannula-Sormunen, M., \& Lehtinen, E. (2015). Modeling the developmental trajectories of rational number concept(s). Learning and Instruction, 37, 14-20. https://doi. org/10.1016/i.learninstruc.2013.12.004

McMullen, J., \& Van Hoof, J. (2020). The role of rational number density knowledge in mathematical development. Learning and Instruction, 65. https:// doi.org/10.1016/i.learninstruc.2019.101228

Moss, J. (2005). Pipes, tubes, and beakers: New approaches to teaching the rational-number system. In M. S. Donovan \& J. D. Bransford (Eds.), How students learn: Mathematics in the classroom (pp. 121-162). Washington, DC: National Academic Press.

Obersteiner, A., Van Dooren, W., Van Hoof, J., \& Verschaffel, L. (2013). The natural number bias and magnitude representation in fraction comparison by expert mathematicians. Learning and Instruction, 28, 64-72. https://doi.org/10.1016/i. learninstruc.2013.05.003

Rossi, S., Vidal, J., Letang, M., Houdé, O., \& Borst, G. (2019). Adolescents and adults need inhibitory control to compare fractions. PsychArchives, 1-43. http://dx.doi.org/10.23668/psycharchives.2381 
Schillemans, V., Luwel, K., Onghena, P., \& Verschaffel, L. (2011). Strategy switch cost in mathematical thinking: Empirical evidence for its existence and importance. Mediterranean Journal for Research in Mathematics Education, 10(1), 1-22.

Schillemans, V., Luwel, K., Onghena, P., Verschaffel, L. (2011). The influence of the previous strategy on individuals' strategy choices. Studia Psychologica, 53(4), 339-350.

Schmidt, J. R., \& De Houwer, J. (2011). Now you see it, now you don't: Controlling for contingencies and stimulus repetitions eliminates the Gratton effect. Acta Psychologica, 138(1), 176-186. https://doi.org/10.1016/i.actpsy.2011.06.002

Schneider, M., \& Siegler, R. S. (2010). Representations of the magnitudes of fractions. Journal of Experimental Psychology: Human Perception and Performance, 36(5), 1227-1238. https://doi. org/10.1037/a0018170

Siegler, R. S., Duncan, G. J., Davis-Kean, P. E., Duckworth, K., Claessens, A., Engel, M., ... \& Chen, M. (2012). Early predictors of high school mathematics achievement. Psychological Science, 23(7), 691697. https://doi.org/10.1177/0956797612440101

Vamvakoussi, X., \& Vosniadou, S. (2010). How many decimals are there between two fractions? Aspects of secondary school students' un- derstanding of rational numbers and their notation. Cognition and Instruction, 28(2), 181-209. https://doi.org/10.1080/07370001003676603

Van Dooren, W., \& Inglis, M. (2015). Inhibitory control in mathematical thinking, problem solving and learning [Special issue]. ZDM Mathematics Education, 47, 713-721. https://doi.org/10.1007/ s11858-015-0715-2

Van Hoof, J., Degrande, T., Ceulemans, E., Verschaffel, L., \& Van Dooren, W. (2018). Towards a mathematically more correct understanding of rational numbers: A longitudinal study with upper elementary school learners. Learning and Individual Differences, 61, 99-108. https://doi. org/10.1016/j.lindif.2017.11.010

Van Hoof, J., Vamvakoussi, X., Van Dooren, W., \& Verschaffel, L. (2017). The transition from natural to rational number knowledge. In D. C. Geary, D. B. Berch, R. Ochsendorf, \& K. M. Koepke (Eds.), Acquisition of complex arithmetic skills and higher-order mathematics concepts (pp. 101-123). London, England: Elsevier.

Verbruggen, F., Liefooghe, B., \& Vandierendonck, A. (2006). The interaction between stop signal inhibition and distractor interference in the flanker and Stroop task. Acta Psychologica, 116(1), 21-37. https://doi.org/10.1016/j.actpsy.2003.12.011 